\title{
Evaluation of BRCA1-related molecular features and microRNAs as prognostic factors for triple negative breast cancers
}

Meriem Boukerroucha ${ }^{1 *+}$, Claire Josse ${ }^{1 * \dagger}$, Sonia ElGuendi ${ }^{1}$, Bouchra Boujemla ${ }^{1}$, Pierre Frères ${ }^{1,2}$, Raphaël Marée ${ }^{4}$, Stephane Wenric ${ }^{1,2}$, Karin Segers ${ }^{3}$, Joelle Collignon ${ }^{2}$, Guy Jerusalem² and Vincent Bours ${ }^{1,3}$

\begin{abstract}
Background: The BRCA1 gene plays a key role in triple negative breast cancers (TNBCs), in which its expression can be lost by multiple mechanisms: germinal mutation followed by deletion of the second allele; negative regulation by promoter methylation; or miRNA-mediated silencing. This study aimed to establish a correlation among the BRCA1-related molecular parameters, tumor characteristics and clinical follow-up of patients to find new prognostic factors.
\end{abstract}

Methods: BRCA1 protein and mRNA expression was quantified in situ in the TNBCs of 69 patients. BRCA1 promoter methylation status was checked, as well as cytokeratin 5/6 expression. Maintenance of expressed BRCA1 protein interaction with BARD1 was quantified, as a marker of BRCA1 functionality, and the tumor expression profiles of 27 microRNAs were determined.

Results: miR-548c-5p was emphasized as a new independent prognostic factor in TNBC. A combination of the tumoral expression of miR-548c and three other known prognostic parameters (tumor size, lymph node invasion and CK 5/6 expression status) allowed for relapse prediction by logistic regression with an area under the curve $(A \cup C)=0.96$.

BRCA1 mRNA and protein in situ expression, as well as the amount of BRCA1 ligated to BARD1 in the tumor, lacked any associations with patient outcomes, likely due to high intratumoral heterogeneity, and thus could not be used for clinical purposes.

Conclusions: In situ BRCA7-related expression parameters could be used for clinical purposes at the time of diagnosis. In contrast, miR-548c-5p showed a promising potential as a prognostic factor in TNBC.

Keywords: BRCA1, TNBC, Breast cancer, miRNA

\section{Background}

Breast cancer susceptibility gene 1 (BRCA1) was the first tumor suppressor gene identified in breast and ovarian cancer. Located on chromosome 17 (17q21), it encodes a multifunctional protein that is involved in several cellular processes such as DNA repair and cell cycle control. BRCA1 is involved in large protein complexes and its

\footnotetext{
*Correspondence: meriem.boukerroucha@doct.ulg.ac.be; c.josse@ulg.ac.be ${ }^{\dagger}$ Equal contributors

'Human Genetics Unit, GIGA-Cancer Research, University of Liège, Liège, Belgium

Full list of author information is available at the end of the article
}

interaction with other proteins, as BARD1, is required for its function.

$B R C A 1$ seems to be associated with the triple negative breast cancer (TNBC) subtype because the histological features and clinical outcomes of TNBC sporadic tumors can be very similar to those found in the tumors of $B R C A 1$ germline mutated patients. The traits that some sporadic cancers share with those occurring in BRCA1 mutation carriers were described and called 'BRCAness' by Turner et al. [1]. In particular, these cancers present a high rate of chromosomal alterations reflecting the absence of the BRCA1 DNA repair function.

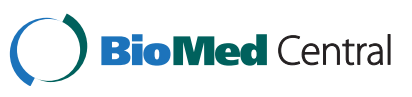

(C) 2015 Boukerroucha et al. Open Access This article is distributed under the terms of the Creative Commons Attribution 4.0 International License (http://creativecommons.org/licenses/by/4.0/), which permits unrestricted use, distribution, and reproduction in any medium, provided you give appropriate credit to the original author(s) and the source, provide a link to the Creative Commons license, and indicate if changes were made. The Creative Commons Public Domain Dedication waiver (http://creativecommons.org/publicdomain/zero/1.0/) applies to the data made available in this article, unless otherwise stated. 
TNBC has a poor prognosis and no targeted therapy is currently available. Because these cancers are heterogeneous in terms of therapeutic response, new therapeutic solutions are being sought. In this context, recent clinical data have shown that BRCA1-associated breast cancers appeared to be more sensitive to platinum agents in neoadjuvant chemotherapy than non-hereditary tumors [2-4]. In contrast, a phase II clinical trial found that poly (ADP-ribose) polymerase (PARP) inhibitors also showed promising activity in BRCA1-mutated breast cancer although there were no response in patients with TNBC regardless of $B R C A 1 / 2$ mutation status [5], and phase III trials are ongoing in $B R C A 1 / 2$-mutated $\mathrm{BC}$ and TNBC [6].

However, it is also becoming clear that germline BRCA1/ 2 mutations are neither necessary nor sufficient for patients to derive benefit from these agents [6]. This variability of response can be explained by different BRCA1 protein expression statuses inside the tumor, as several cases can be met : (i) germline BRCA1 is mutated in one allele, and the second is lost; thus, BRCA1 tumoral expression is missing [7, 8] (ii) germline BRCA1 is mutated in one allele, and the second is still active, so tumoral BRCA1 expression is normal; (iii) germline $B R C A 1$ is mutated, but reversal somatic mutation occurs, and BRCA1 tumoral expression is restored, leading to PARP inhibitor treatment resistance [9, 10]; (iv) germline $B R C A 1$ is normal, but tumoral expression is lost by promoter hypermethylation [11]; and (v) germline BRCA1 is normal, but tumoral expression is lost by post-transcriptional regulation, such as by miRNAs [12]. One could expect a greater likelihood of response for patients treated with platinum compounds or PARP inhibitors only if BRCA1 protein tumoral expression were lost. As a consequence, better characterization of BRCA1 expression status in TNBC would provide important knowledge to improve chemotherapy choices.

MicroRNAs are small non-coding RNAs that bind to the 3' untranslated (3'UTR) region of target messenger RNAs (mRNAs), and they are known to regulate gene expression. They are deregulated in breast cancers: some of them are known to be oncogenic, and others are known as tumor suppressors. MiRNAs participate in a variety of biological processes, such as the immune response, as well as proliferation and metastasis, which are hallmarks of cancer $[13,14]$. Many studies have implicated miRNAs in chemotherapy resistance, such as to cisplatin [15], and some of them have been used as prognostic biomarkers [16-18]. Moreover, some miRNAs could target BRCA1 mRNA expression, and, at the same time, their expression was affected by BRCA1 protein [12, 19-21].

Currently, the number of conventional breast cancer prognostic factors is limited (tumor size, histology and grade, hormone receptors status, lymph nodes invasion, proliferative index [Ki67], and tumor-infiltrating lymphocytes, as well as the age of the patient), and their use does not allow for accurate prediction of treatment resistance or relapse in TNBC. Defining new molecular prognostic factors to refine TNBC classification would be useful in facilitating a more adapted chemotherapy choice.

In this context, we quantified molecular parameters focusing on the BRCA1 gene expression regulation and function (BRCA1 promoter methylation, BRCA1 in situ mRNA expression, BRCA1 in situ protein expression and BRCA1 in situ interaction with BARD1) in 69 TNBC tumors from patients. The expression of 27 tumoral miRNAs was also measured. Those molecular parameters were associated with progression-free survival in uni- and multivariate statistical analyses to determine new prognostic factors.

\section{Methods}

More detailed protocols are available in Additional file 1.

\section{Ethical statement}

Ethical approval was obtained from the local institutional ethics board (Comité d'éthique hospitalo-facultaire universitaire de Liège) in compliance with the Helsinki declaration. All of the patients were recruited on the basis of an opt-out methodology.

\section{Patient and sample collection and study design}

This retrospective study was performed on 69 formalinfixed paraffin embedded (FFPE) tumoral samples obtained from the Liege University Biobank. The tissues stored in this biobank are available on condition that the study has received the consent of a local or external ethical board. The tumors were collected from 1999 to 2010, with a median follow-up of 11 years. The essential elements of "Reporting recommendations for tumor marker prognostic studies (REMARK)" were followed [22].

The clinicopathological characteristics of the patients are summarized in Table 1.

A summary of the experimental design and the number of samples included in each type of analysis are shown in Fig. 1.

\section{DNA and RNA extraction}

DNA and RNA extraction was performed using an All Prep DNA/RNA FFPE extraction kit from Qiagen (Belgium) according to manufacturer protocol. Multiplex PCR for increasing the size amplicons of a house keeping gene was performed to assess the nucleic acid quality, as described by van Beers et al. [23]. 
Table 1 Patient clinicopathological characteristics

\begin{tabular}{|c|c|}
\hline & $n=69$ \\
\hline \multicolumn{2}{|l|}{ Age (year) } \\
\hline median & 56 \\
\hline range & $27-89$ \\
\hline \multicolumn{2}{|l|}{ Tumor size (mm) } \\
\hline$<20$ & 23 \\
\hline$\geq 20$ & 35 \\
\hline unknown & 11 \\
\hline \multicolumn{2}{|l|}{ Lymph node invasion } \\
\hline yes & 15 \\
\hline no & 38 \\
\hline unknown & 16 \\
\hline \multicolumn{2}{|l|}{ Ki 67 (\%) } \\
\hline$<20$ & 11 \\
\hline$\geq 20$ & 52 \\
\hline unknown & 6 \\
\hline \multicolumn{2}{|l|}{ Histology } \\
\hline IDC & 47 \\
\hline other & 19 \\
\hline unknown & 3 \\
\hline \multicolumn{2}{|l|}{ Bloom } \\
\hline 1 & 6 \\
\hline$\|$ & 7 \\
\hline III & 53 \\
\hline unknown & 3 \\
\hline \multicolumn{2}{|l|}{ Molecular subtype } \\
\hline ck5/6 +, ER-, Her2- & 30 \\
\hline ck5/6 -, ER-, Her2- & 32 \\
\hline unknown & 7 \\
\hline \multicolumn{2}{|l|}{ Relapse } \\
\hline yes & 24 \\
\hline no & 45 \\
\hline
\end{tabular}

\section{BRCA1 promoter methylation}

The methylation status of $B R C A 1$ promoter was assessed by methylation-specific PCR (MSP), as described by Esteller et al. [24].

\section{BRCA1 mRNA expression}

The mRNA expression was assessed by in situ hybridization using RNAscope technology (ACD) (Bioke, the Netherlands) for FFPE samples, as described in our previous work [25]. Signal quantification was performed using the Cytomine application (http://www.cytomine.be/, Marée et al. 2013) [26]. BRCA1 mRNA expression was expressed as a percentage of the median expression value measured in the whole group.

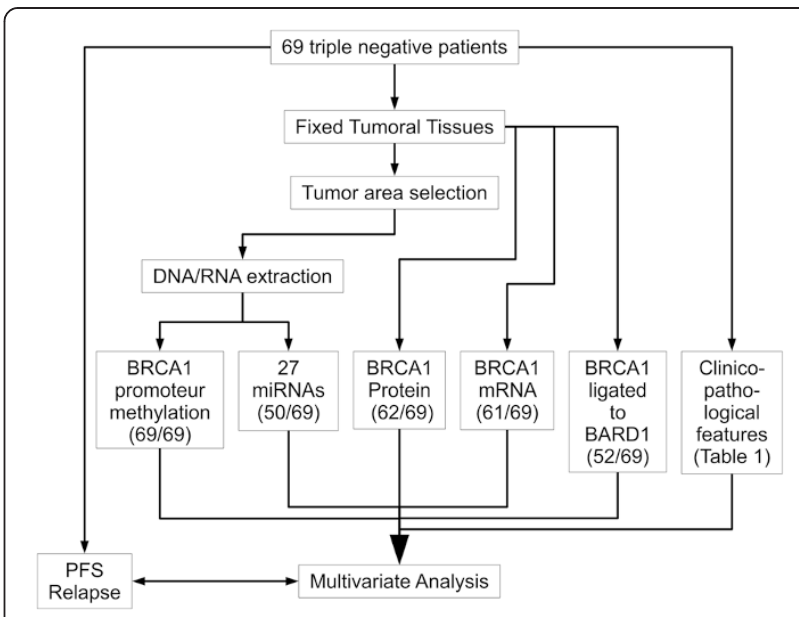

Fig. 1 Schematic representation of the study

BRCA1 protein expression and interaction with BARD1

BRCA1 expression level and interaction with BARD1 were assessed by proximity ligation assay (Duolink in situ detection reagents-Sigma, Belgium), as described in [25] and in Additional file 1. Two antibodies raised against BRCA1 were used for the whole-length protein detection assays, and one antibody against BRCA1 and a second against BARD1 were used for interaction assays. The amount of BRCA1 protein and the amount of BRCA1-ligated to BARD1 were expressed as a percentage of their respective median expression values measured in the whole group.

\section{Tumoral miRNA expression assessment}

A total of 27 miRNAs were quantified by RT-qPCR in tumors using miRCURY LNA ${ }^{\mathrm{TM}}$ Universal RT microRNA PCR assays from Exiqon (Denmark), according to the manufacturer's instructions. Those miRNAs were chosen because: (i) their expression was reported in the literature to be related to the survival of breast cancer patients; (ii) they are known to be expressed in lymphoid cells and to reflect the lymphoid invasion of the tumor; or (iii) they were emphasized in our previous work (unpublished results). The miRNAs quantified, their sequences and the reasons for choosing them are listed in Additional file 2.

Quantification was realized using standard curve method. Normalization was performed using the geometric mean of five endogenous control genes. The miRNA amounts were expressed in percentages relative to the median expression value of the whole group.

\section{Statistical analysis}

Statistical analysis were performed with SPSS software (version 20.0: IBM SPSS), and checked with $\mathrm{R}$ software (version 3.1.0). Some of the graphs were drawn with GraphPad Prism software, version 5. 


\section{Results}

\section{Quantification of in situ BRCA1 mRNA and protein expression}

To assess the BRCA1 expression status inside the tumors, the amount of BRCA1 protein was first measured by proximity ligation assays (PLAs) in fixed TNBC tissues. Representative in situ BRCA1 protein expression is shown in Fig. 2a. As a second step, the BRCA1 mRNA expression level was visualized and quantified in the same tissues, by in situ hybridization (Fig. 2b).

The most striking observation was that the staining for both mRNA and protein is heterogeneous across the tumor: some areas strongly expressed BRCA1 and others only faintly, as illustrated in the two magnified subzones. The staining was restricted to epithelial cells.
Univariate analyses showed that neither BRCA1 protein nor mRNA expression was associated with progressionfree survival (PFS) (Fig. 2c). The entire dataset and all of the univariate analyses performed in this study are available in Additional files 3 and 4.

\section{Quantification of in situ BRCA1-BARD1 interaction}

Proximity ligation assays were performed to quantify the in situ interaction of BRCA1 with its interacting protein, BARD1. Statistical analyses revealed that the percentage of BARD1-ligated BRCA1 was correlated with BRCA1 protein and mRNA expression. However, no association was observed with PFS in univariate analysis (Fig. 2c).
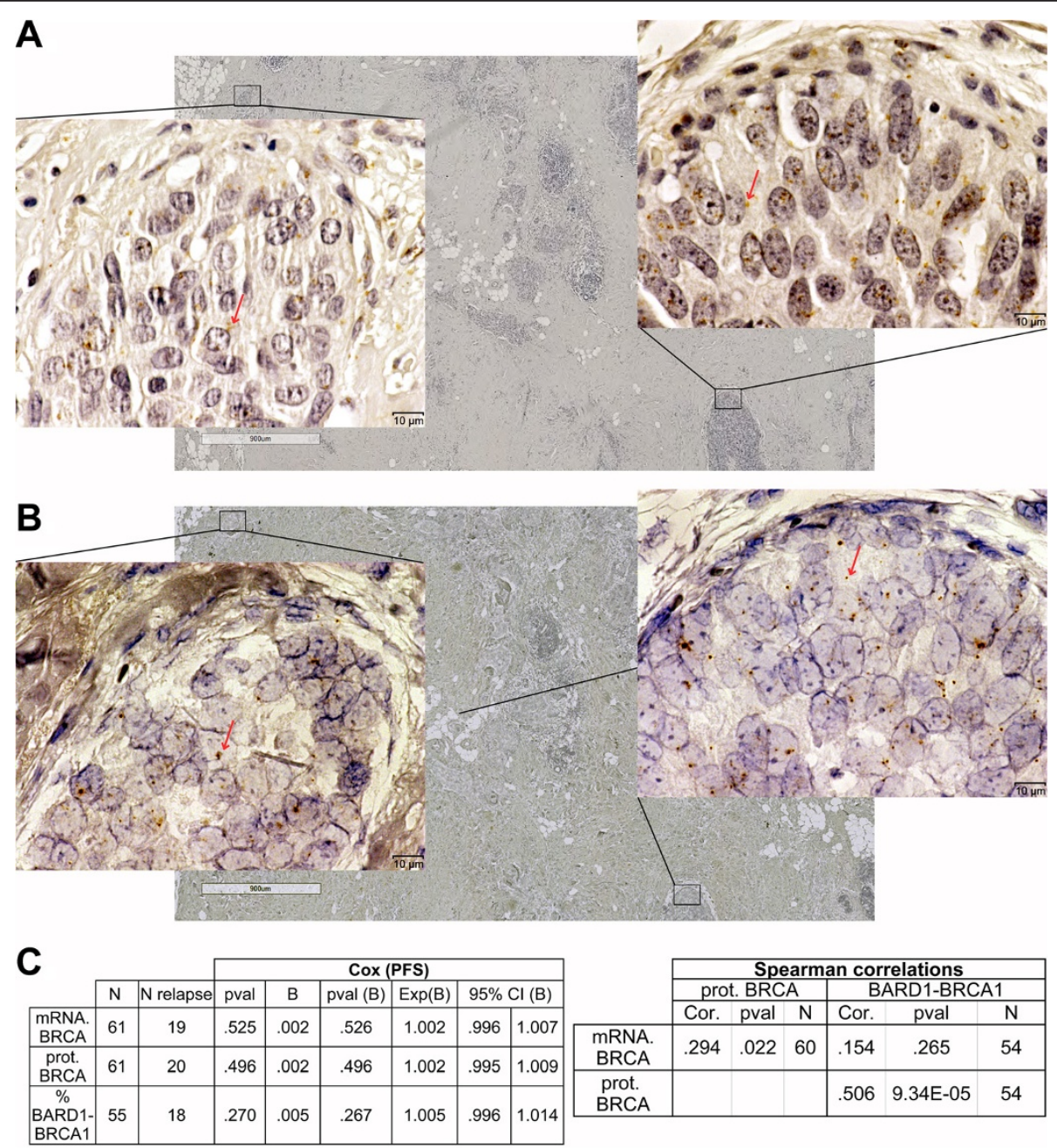

Fig. 2 In situ BRCA1 expression in TNBC tumors. a Proximity ligation assay showing a representative BRCA1 protein expression across the tumor. Two different subzones were magnified to illustrate high and faint expression. $\mathbf{b}$ In situ hybridization assay showing BRCA1 mRNA expression across the same tumor and subzones used for protein detection. In both cases, high heterogeneity of the localization of expression is observed. c. Cox univariate regression and correlation analyses of BRCA1 expression relative to patient clinicopathological features. No relationship of BRCA1 expression with patient outcome was observed 


\section{$B R C A 1$ promoter methylation and survival}

The methylation status of $B R C A 1$ promoter was checked by methylation-specific PCR in tumoral DNA extracted from fixed TNBC tissues. Twenty-seven of the 69 patients (39\%) carried a methylated $B R C A 1$ promoter, but we did not observe any associations of $B R C A 1$ promoter methylation with patient outcomes or with $B R C A 1$ mRNA expression (Additional file 5). However, an expected negative correlation was observed between methylation and protein expression in the infiltrating ductal carcinoma sub-group.

\section{Micro-RNA profiling in tumors}

The tumoral expression of 27 miRNAs was quantified by RT-qPCR in RNA extracted from fixed TNBC tissues.

Spearman's correlations were calculated of the studied miRNAs and BRCA1 mRNA with protein expression, BARD1-BRCA1 interaction, and promoter methylation status. The entire dataset is presented in Additional file 3. BRCA1 protein expression was positively correlated with miR-143-3p ( $p=0.033)$, miR-205-5p $(p=0.030)$, miR-21-5p $(p=0.017)$, and miR-142-5p $(p=0.011)$. In contrast, no correlation was noted with $B R C A 1$ mRNA. Promoter methylation was negatively correlated with miR-21-5p $(p=0.024)$ and positively correlated with miR-197-3p ( $p=0.019)$.

Univariate Cox regression analyses were also conducted to emphasize the associations of miRNA expression with patient outcomes (Table 2 and Additional file 4). High expression of miR-210, miR-205-5p, miR-484, and miR-93-5p were significantly associated with an increased risk of relapse, and miR-342-3p, reflecting lymphoid cell infiltration [27], was associated with a good prognosis (Table 2).

\section{Prediction of relapse using multivariate analysis}

Univariate Cox proportional hazards regression analyses were first conducted to evaluate the association of clinicopathological factors with patient PFS (Additional file 4). Node invasion, cytokeratin five and six expression, bloom $=3$ and the size of the tumor are associated with relapse.

In multivariate Cox analysis, three parameters remained as independent prognostic factors: node invasion status, tumor size, and the expression of miR-548c-5p (node invasion: $\operatorname{Exp}(\mathrm{B})$ : 16.576; CI: 2.876-95.538; $p$-val: 0.002-tumor size: $\operatorname{Exp}(\mathrm{B}): 1.065$; CI: $1.027-1.105$; $p$-val: $0.001-\mathrm{miR}-$ 548c-5p: $\operatorname{Exp(B):~0.993;~CI:~0.987-0.999;~p-val:~0.023).~}$

An outcome prediction model was built by binomial logistic regression. The best prediction model used node invasion, the size of the tumor, cytokeratin 5/6 expression status and miR-548c-5p. Because the first three variables were already known to be prognostic factors, we compared the performances of two models, containing or not containing the miR-548c-5p expression variable, to evaluate the improvement of the prediction of relapse by this miRNA (Fig. 3). The addition of miR-548c-5p statistically improved the model (Chi-square $p$-val $=0.00144$ ). A ROC curve corresponding to the probability of relapse for each patient, calculated by these two models, is shown in Fig. 3a. The use of miR-548c-5p expression allowed for the improvement of the AUC from 0.854 (CI:0.713 to 0.996)

Table 2 Univariate Cox analysis

\begin{tabular}{|c|c|c|c|c|c|c|c|c|}
\hline Variable & $N$ total & $N$ relapse & Global pval & $B$ & Sign & $\operatorname{Exp}(B)$ & $95 \% \mathrm{Cl}$ & \\
\hline miR-210 & 49 & 20 & 0.00 & .004 & .000 & 1.004 & 1.002 & 1.007 \\
\hline miR-205-5p & 49 & 20 & 0.00 & .003 & .002 & 1.003 & 1.001 & 1.005 \\
\hline Node & 53 & 21 & 0.00 & -1.344 & .003 & .261 & .108 & .630 \\
\hline miR-484 & 49 & 20 & 0.01 & .004 & .015 & 1.004 & 1.001 & 1.008 \\
\hline CK & 61 & 20 & 0.02 & 1.106 & .024 & 3.023 & 1.159 & 7.881 \\
\hline miR-93-5p & 49 & 20 & 0.02 & .003 & .019 & 1.003 & 1.001 & 1.006 \\
\hline Bloom = 3 & 65 & 23 & 0.03 & 1.963 & .055 & 7.117 & .957 & 52.955 \\
\hline miR-342-3p & 49 & 20 & 0.04 & -.005 & .049 & .995 & .990 & 1.000 \\
\hline Size & 57 & 18 & 0.05 & .016 & .055 & 1.016 & 1.000 & 1.032 \\
\hline Age & 68 & 24 & 0.07 & .025 & .070 & 1.025 & .998 & 1.053 \\
\hline Bloom = 1 & 65 & 23 & 0.08 & -3.195 & .262 & .041 & .000 & 10.892 \\
\hline miR-146a & 49 & 20 & 0.09 & -.004 & .093 & .996 & .992 & 1.001 \\
\hline miR-143-3p & 49 & 20 & 0.11 & .003 & .116 & 1.003 & .999 & 1.007 \\
\hline miR-155-5p & 49 & 20 & 0.11 & -.003 & .123 & .997 & .993 & 1.001 \\
\hline miR-150-5p & 49 & 20 & 0.12 & -.004 & .151 & .996 & .991 & 1.001 \\
\hline miR-142-3p & 49 & 20 & 0.18 & -.003 & .195 & .997 & .993 & 1.002 \\
\hline $\operatorname{miR}-548 c-5 p$ & 49 & 20 & 0.19 & -.001 & .196 & .999 & .997 & 1.001 \\
\hline miR-374a-5p & 49 & 20 & 0.20 & -.005 & .200 & .995 & .988 & 1.003 \\
\hline
\end{tabular}




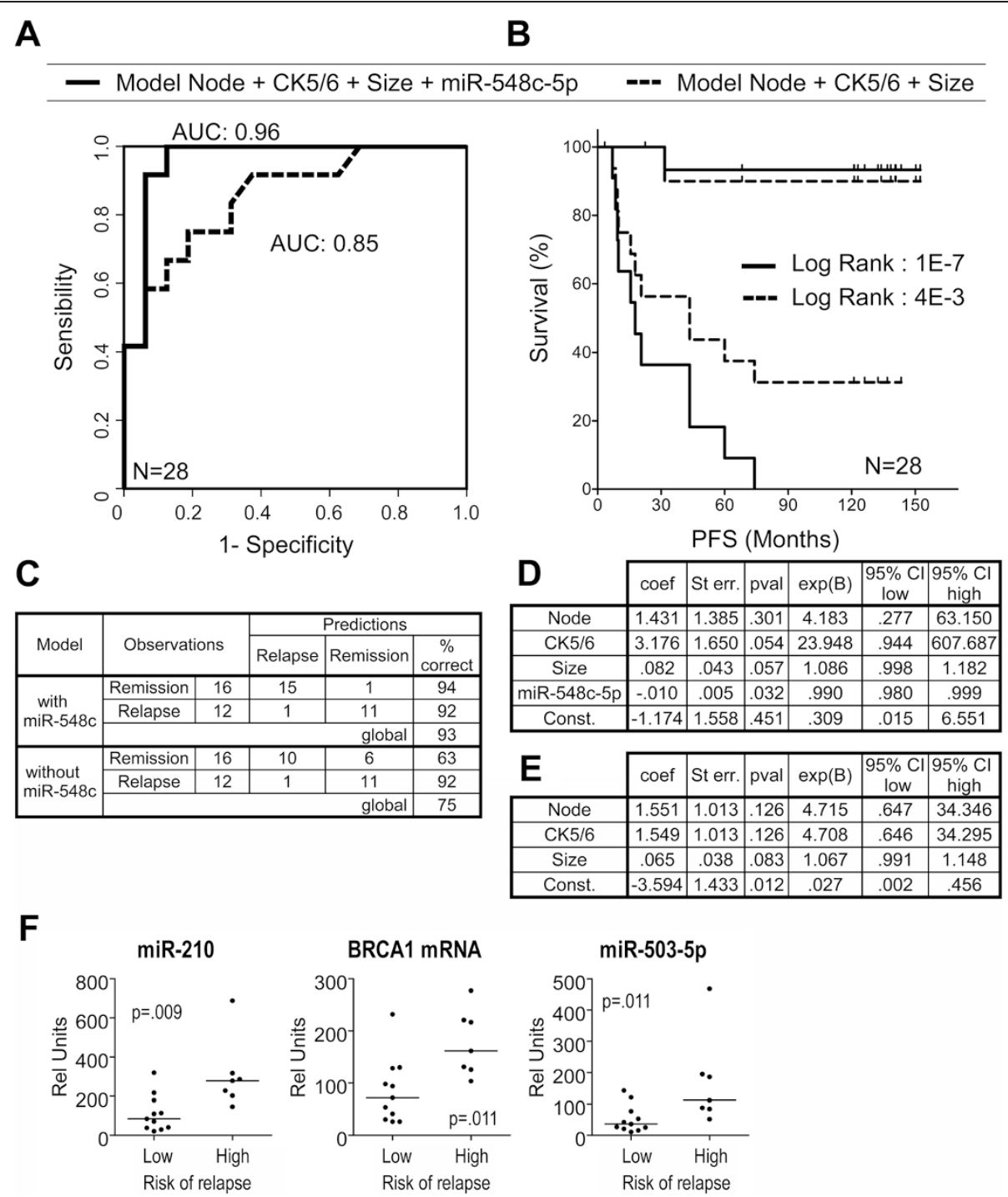

Fig. 3 miR-548c-5p as factor in relapse prediction model. Performances of two models are compared to measure the improvement of relapse prediction by the inclusion of the miR-548c-5p as a 4th variable, with the first three variables being node invasion, CK5/6 expression, and tumor size. a Comparison of ROC curves computed with the relapse probability calculated by the model including miR-548c-5p (solid line) and without miR-548c-5p (dash line). b Patients were classified in two groups: high and low risk of relapse, according to the threshold needed to obtain $90 \%$ sensitivity in relapse prediction. Comparison of Kaplan-Meier curves computed with the patient group affectation calculated by the model including miR-548c-5p (solid line) and without miR-548c-5p (dash line). c Classification performances of the two models at thresholds fixed to obtain $90 \%$ sensitivity in relapse detection. d Coefficient and odds ratio of the model including miR-548c-5p and e without miR-548c-5p. f Comparative expression levels of miR-210, miR-503-5p and BRCA1 mRNA in the patients with $<10 \%$ probability of relapse (remission) and $>90 \%$ probability of relapse (relapse). These probabilities were calculated by the prediction model including miR-548c-5p

to 0.958 (CI:0.883-1.000)(Table 3). Thresholds for both models were chosen to fix relapse detection sensitivity at $90 \%$. Using these thresholds, the patients were assigned by each model into two risk groups: low or high risk of relapse. Kaplan-Meier PFS curves were generated using these effect groups for both models, and they are shown in Fig. 3b. Classification performances of the compared models are presented in Fig. 3c, and metrics are shown in Fig. 3d (with miR-548c) and 3e (without miR-548c) and in Table 3.
Interestingly, a comparison of two groups of patients presenting with extreme relapse probabilities $(<10 \%$ and $>90 \%$ ), calculated by the predicting model including miR-548c, showed that patients with poor prognoses present higher expression of miR-503-5p, miR-210 and BRCA1 mRNA.

In contrast, the addition of $B R C A 1$-related parameters (mRNA, protein and BARD1 ligated to BRCA1) to the same three conventional prognostic factors (node invasion, 
Table 3 Performances metrics of the logistical regression models

\begin{tabular}{|c|c|c|c|c|c|c|c|c|c|c|}
\hline \multirow[b]{2}{*}{ Variables } & \multicolumn{3}{|c|}{ Global model performances } & \multicolumn{4}{|l|}{$R O C$} & \multicolumn{3}{|c|}{ Hosmer-Lemeshow } \\
\hline & pval & $-2 L L$ & R2 Nagelkerke & $A \cup C$ & SE & Cl: Min & $\mathrm{Cl}:$ Max & Chi2 & $d d l$ & pval \\
\hline Node, Tumor Size, CK5/6, miR-548c-5p & $1.5 \mathrm{E}-4$ & 15.570 & .745 & .96 & .038 & .883 & 1.000 & 5.08 & 7 & .65 \\
\hline $\begin{array}{l}\text { Node, Tumor Size, CK5/6, prot BRCA1, mRNA BRCA1, } \\
\text { BARD1 ligated BRCA1 }\end{array}$ & .009 & 21.979 & .590 & .90 & .065 & .773 & 1.000 & 11.45 & 8 & .18 \\
\hline Node, Tumor Size, CK5/6 & .006 & 25.722 & .484 & .85 & .072 & .713 & .996 & 2.96 & 7 & .89 \\
\hline
\end{tabular}

tumor size and CK5/6 expression) did not improve the model performances (Fig. 4 and Table 3).

\section{Discussion}

An accurate technique to determine BRCA1 tumoral expression status in TNBC would allow for informed decision and choosing platinum derivatives or PARP inhibitor treatments, because hypersensitivity to these agents has been described in cases of loss of BRCA1 expression $[3,5]$. Thus, we developed alternative techniques to evaluate, in tumors, the expression status of BRCA1 at three different levels: mRNA, protein, and maintenance of
BRCA1 interaction with BARD1. This multiple approach presented the advantage of incorporating different types of information, allowing for cross-control, and offering the possibility of combining the data. Several BRCA1 studies have described mRNA expression using RT-qPCR or protein expression by immunohistochemistry, but studies describing both mRNA and protein expressions has been very rare [28], despite BRCA1 expression being known to be subjected to multiple regulations [19]. The commercially available antibodies directed against BRCA1 lack the specificity required to identify the BRCA1 protein for clinical purpose because no immunohistochemical

A

B
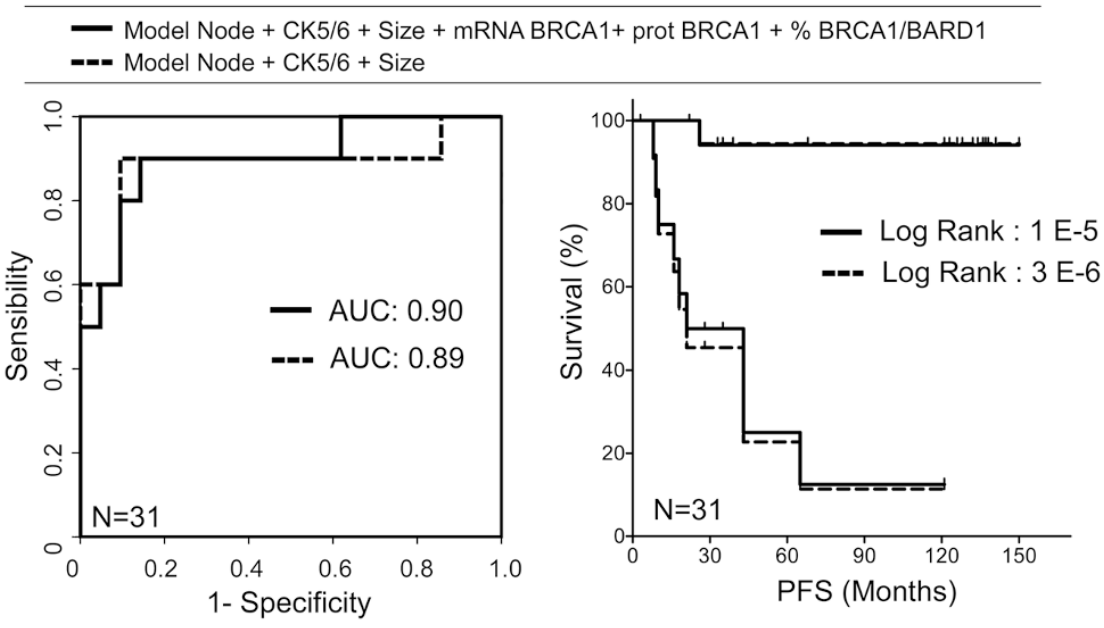

\begin{tabular}{|c|c|c|c|c|c|}
\hline & \multirow{2}{*}{\multicolumn{2}{|c|}{ Observations }} & \multicolumn{3}{|c|}{ Predictions } \\
\hline & & & Relapse & Remission & $\%$ \\
\hline \multirow{3}{*}{$\begin{array}{l}\text { with } \\
\text { BRCA1 } \\
\text { param. }\end{array}$} & Remission & 21 & 18 & 3 & 86 \\
\hline & Relapse & 10 & 1 & 9 & 90 \\
\hline & & & & global & 87 \\
\hline \multirow{3}{*}{$\begin{array}{l}\text { without } \\
\text { BRCA1 } \\
\text { param. }\end{array}$} & Remission & 21 & 19 & 2 & 90 \\
\hline & \begin{tabular}{|l|} 
Relapse \\
\end{tabular} & 10 & 1 & 9 & 90 \\
\hline & \multicolumn{4}{|r|}{ global } & 90 \\
\hline
\end{tabular}

\begin{tabular}{|c|c|c|c|c|c|c|}
\cline { 2 - 7 } $\mathbf{D}$ & coef & St err. & pval & $\exp (\mathrm{B})$ & $\begin{array}{c}95 \% \text { Cl } \\
\text { low }\end{array}$ & $\begin{array}{c}95 \% \mathrm{Cl} \\
\text { high }\end{array}$ \\
\hline Size & .062 & .031 & .049 & 1.064 & 1.000 & 1.132 \\
\hline Node & 2.330 & 1.250 & .062 & 10.282 & .888 & 119.066 \\
\hline CK5/6 & 3.408 & 1.665 & .041 & 30.203 & 1.155 & 789.957 \\
\hline mRNA BRCA1 & -.011 & .009 & .219 & .989 & .971 & 1.007 \\
\hline prot BRCA1 & .001 & .010 & .950 & 1.001 & .981 & 1.021 \\
\hline \%BRCA1-BARD1 & .019 & .014 & .164 & 1.019 & .992 & 1.047 \\
\hline Const. & -7.042 & 2.920 & .016 & .001 & .000 & .268 \\
\hline
\end{tabular}

Fig. 4 BRCA1 expression as factor in relapse prediction models. Performances of two models are compared: the first model (solid line) includes BRCA1 expression parameters mRNA, protein expression and BARD1-ligated BRCA1, in addition to the three previously used conventional prognostic factors for breast cancer: tumor size, node invasion, CK5/6 expression. The second model (dash line) is composed of the three conventional prognostic factors only. a Comparison of ROC curves computed with the relapse probability calculated by the BRCA1-related model (solid line) and the three conventional prognostic factor model (dash line). b Patients were classified in two groups: good or bad prognosis, according to the threshold needed to obtain $90 \%$ sensitivity in relapse detection. Comparison of Kaplan-Meier curves computed with the patient group affectation calculated by the two models is represented. c. Classification performances of the two models at threshold fixed to obtain $90 \%$ sensitivity in relapse detection. $\mathbf{d}$. Coefficient and odds ratio of the model including BRCA1 expression parameters 
(IHC) differences in BRCA1 protein expression were found between cases with and without BRCA1 germline mutations by Pérez-Vallés and colleagues [29]. To improve the sensitivity and specificity of the BRCA1 detection compared with IHC, we used proximity ligation assays with two primary antibodies against the $\mathrm{N}$ - and $\mathrm{C}$-terminus domains of BRCA1. The second advantage of this technique was that it only allowed for the measurement of the full-length proteins. BRCA1 must be ligated to its interacting protein BARD1 to repair DNA. Some BRCA1 variants, such as splicing variants [29], can be expressed in the tumor but can lose their interaction with their partners. To obtain a reflection of BRCA1 function maintenance in tumors, proximity ligation assay were performed to visualize the portion of BRCA1 ligated to BARD1.

Although the three levels of BRCA1 tumoral expression were correlated inside the same tumor, highly heterogenous intra-tumoral expression was observed, hampering accurate quantification. The lack of correlation between PFS and BRCA1 expression was probably a consequence of this high variability. We concluded that none of these three facets of the BRCA1 tumoral expression could be used for clinical decision purposes.

The TNBC cohort that we explored included six patients with a known germline BRCA1 mutation. However, no significant differences in BRCA1 expression at the levels of mRNA, protein, or ligation to BARD1 were observed in these cases, probably due to the small number of patients. Interestingly, two of these six BRCA1 mutated patients also presented a methylated form of the $B R C A 1$ promoter, although Lips et al. described these events as mutually exclusive [30]. This combination of events could increase the risk of breast cancer because these patients are also the two youngest who developed breast cancer in our cohort of 69 patients, but this possibility will need to be confirmed on a larger cohort. The work of Ertuk and Cecener also stated that miRNAs expression can be different in BRCA1 mutated or normal TNBC tumors [31]. However, we could not observe similar effect, probably due to the small number of patients.

Statistical multivariate analysis demonstrated that miR548c-5p was an independent prognostic factor for breast cancer. Patients with a good prognosis presented higher intratumoral expression of this microRNA. Although implicated in multiple biological processes including cancer, no role for miR-548c-5p has ever been reported in the breast cancer field. Mir-548 is a large, poorly conserved primate-specific miRNA gene family. Sixty-nine human mir-548 genes are located on almost all human chromosomes and its widespread distribution pattern and specific sequence indicate its evolutionary origin from the MADE1 transposable element $[32,33]$. There are more than 3500 putative mir-548 target genes, but none have been experimentally demonstrated for miR-548c-5p.
The measurement of tumoral miR-548c-5p expression levels in combination with three conventional breast cancer prognostic factors (node invasion, tumor size and cytokeratin 5/6 expression), allowed for the relapse prediction of patients with an AUC $=0.96$. A study in a larger cohort would be needed to confirm this observation, and to determine whether quantification of this microRNA expression in the tumor could be used to steer patients with poor predicted prognosis toward alternative chemotherapies.

We also showed that patients with poor predicted prognoses calculated by this model presented higher expression of miR-210, miR-503-5p and BRCA1 mRNA. Indeed, high miR-210 expression has already been reported by other teams to be correlated with relapse and short survival $[19,34]$. miR-503-5p was already emphasized in our previous work: this microRNA is highly expressed in endothelial cells and, can be secreted in exosomes and transferred into breast cancer cell lines to inhibit tumor growth by targeting CCND2 and CCND3 [35]. Moreover, neoadjuvant chemotherapy for breast cancer leads to increased plasma levels of miR-503, as also observed for miR-34a, which could be implicated in the anti-tumor effects of chemotherapy in breast cancer patients [35, 36]. Concerning the higher expression of BRCA1 mRNA observed in the poor-prognosis tumors, we could hypothesize that patients expressing high levels of $B R C A 1$ would present a lower response to chemotherapy because TNBC BRCA1 mutated patients are known to respond better to chemotherapy [37].

MiR-484 was reported by Dvinge et al. as a good potential housekeeping microRNA in breast cancer because its expression was homogenous among samples in all breast cancers subtypes [27]. However, Cox univariate analysis showed that high miR-484 expression was associated with a bad prognosis in our TNBC cohort. Volinia et al. also reported such an association [18]. In a high-throughput study aiming at better defining miRNAmRNA interaction, $B R C A 1$ was identified as interacting with miR-484. However, we did not observed any inverse correlation between those two parameters. Although, miR-484 expression was strongly associated with two other poor-prognosis miRNAs: miR-205 (Rho Spearman: 0.4, $p$-val :0.003) and miR-93 (Rho Spearman : 0.52, $p$ $\mathrm{val}=0.0001)$, the Diana MiRPath database did not present any experimentally demonstrated common target gene of the three miRNAs [38].

\section{Conclusions}

BRCA1 was expressed in a spatially heterogeneous manner in TNBC, making very difficult any study correlating its expression or activity with prognosis. However, this study emphasized miR-548c-5p tumoral expression as a new independent prognostic factor that could improve the performance of relapse prediction models based on 
node invasion, tumor size and cytokeratin five and six expression status.

\section{Additional files}

\section{Additional file 1: Detailed description of the protocols used in the \\ study. (DOCX $24 \mathrm{~kb}$ )}

Additional file 2: List of the microRNAs quantified by RT-qPCR, their sequences and the reasons for their choice for the study. The most significant MSigDB Canonical Pathways affected by each microRNAs are mentioned as provided by the StarBase v2.0 web server issue [39]. (DOCX 58 kb)

\section{Additional file 3: Table of the entire variable and observation} dataset. (XLS $53 \mathrm{~kb}$ )

Additional file 4: Results of the univariate analyses performed on the dataset. (XLS $109 \mathrm{~kb}$ )

Additional file 5: BRCA1 promoter methylation. A. Kaplan Meier curves showing the lack of relationship of BRCA1 promoter methylation with progression-free survival. B. Cox univariate regression and correlation analyses of BRCA1 promoter methylation relative to patient clinicopathological features. (TIFF $307 \mathrm{~kb}$ )

\section{Competing interests}

The authors declare that they have no competing of interests.

\section{Authors' contributions}

Conception and design: MB, CJ, GJ, and VB; Development and methodology: $\mathrm{MB}, \mathrm{CJ}, \mathrm{GJ}$, and VB; Acquisition of data: MB, CJ, SE, BB, PF, RM, KS, and JC; Analysis and interpretation of data: MB, CJ, SE, KS, and SW; Writing, review and revision of the manuscript: MB, CJ, GJ, and VB; Study Supervision: MB, CJ, GJ, and VB. All authors read and approved the final manuscript.

\section{Acknowledgments}

We first thank the patients. We also thank the Biobanque of Liège University Hospital, the GIGA-Immunohistology platform, the GIGA-Bioinformatics platform, and the GIGA-Imaging platform. We thank also Hélène Schroeder, Corinne Fasquelle, Fabienne Perin, Ingrid Struman, Philippe Delvenne, Christophe Poulet, Amaury Bynens, Gustavo Moraes, Laurent Schoysman, Jérôme Kroonen, Jérôme Thiry and Tiberio Sticca. We thanks American Journal Expert for language revision.

Funding was obtained from the following institutions: Fonds de la Recherche Scientifique : Télévie (MB), Projet de recherche fundings (SW) and Candidat Spécialiste Doctorant (PF); Centre-Anti-Cancéreux (MB); Fond d'Investissement à la recherche Scientifique du Centre Universitaire Hospitalier de Liège (manuscript preparation); Région Wallonne (CJ); Belgian Fundation against Cancer (CJ; BB and manuscript preparation); and the Fonds européen de développement économique et régional (RM).

\section{Author details}

${ }^{1}$ Human Genetics Unit, GIGA-Cancer Research, University of Liège, Liège, Belgium. ${ }^{2}$ Medical Oncology Department, University of Liège and CHU de Liège, Liège, Belgium. ${ }^{3}$ Center of Genetics, CHU de Liège, Liège, Belgium. ${ }^{4}$ GIGA-bioinformatics platform, University of Liège, Liège, Belgium.

Received: 30 April 2015 Accepted: 8 October 2015

Published online: 21 October 2015

\section{References}

1. Turner N, Tutt A, Ashworth A. Hallmarks of "BRCAness" in sporadic cancers. Nat Rev Cancer. 2004;4(October):814-9.

2. von Minckwitz G, Schneeweiss A, Loibl S, Salat C, Denkert C, Rezai M, et al. Neoadjuvant carboplatin in patients with triple-negative and HER2-positive early breast cancer (GeparSixto; GBG 66): a randomised phase 2 trial. Lancet Oncol. 2014:15:747-56.

3. Byrski T, Huzarski T, Dent R, Marczyk E, Jasiowka M, Gronwald J, et al. Pathologic complete response to neoadjuvant cisplatin in BRCA1-positive breast cancer patients. Breast Cancer Res Treat. 2014;147:401-5.
4. Sikov WM, Berry DA, Perou CM, Singh B, Cirrincione CT, Tolaney SM, et al. impact of the addition of carboplatin and/or bevacizumab to neoadjuvant once-per-week paclitaxel followed by dose-dense doxorubicin and cyclophosphamide on pathologic complete response rates in stage II to III triple-negative breast cancer: CALGB 40603 (a. J Clin Oncol. 2015;33:13-21.

5. Tutt A, Robson M, Garber JE, Domchek SM, Audeh MW, Weitzel JN, et al. Oral poly(ADP-ribose) polymerase inhibitor olaparib in patients with BRCA1 or BRCA2 mutations and advanced breast cancer: a proof-of-concept trial. Lancet. 2010;376:235-44.

6. Scott CL, Swisher EM, Kaufmann SH. Poly (ADP-ribose) polymerase inhibitors: recent advances and future development. J Clin Oncol. 2015;33:1397-406.

7. Osorio A, de la Hoya M, Rodríguez-López R, Martínez-Ramírez A, Cazorla A, Granizo JJ, et al. Loss of heterozygosity analysis at the BRCA loci in tumor samples from patients with familial breast cancer. Int J Cancer. 2002;99:305-9.

8. Cornelis RS, Neuhausen SL, Johansson O, Arason A, Kelsell D, Ponder BA, et al. High allele loss rates at 17q12-q21 in breast and ovarian tumors from BRCAl-linked families. The breast cancer linkage consortium. Genes Chromosomes Cancer. 1995;13:203-10.

9. Norquist B, Wurz KA, Pennil CC, Garcia R, Gross J, Sakai W, et al. Secondary somatic mutations restoring BRCA1/2 predict chemotherapy resistance in hereditary ovarian carcinomas. J Clin Oncol. 2011;29:3008-15.

10. Barber LJ, Sandhu S, Chen L, Campbell J, Kozarewa I, Fenwick K, et al. Secondary mutations in BRCA2 associated with clinical resistance to a PARP inhibitor. J Pathol. 2013;229:422-9.

11. Veeck J, Ropero S, Setien F, Gonzalez-Suarez E, Osorio A, Benitez J, et al. BRCA1 CpG island hypermethylation predicts sensitivity to poly(adenosine diphosphate)-ribose polymerase inhibitors. J Clin Oncol. 2010;28:e563-4. author reply e565-6.

12. Tan X, Peng J, Fu Y, An S, Rezaei K, Tabbara S, et al. miR-638 mediated regulation of BRCA1 affects DNA repair and sensitivity to UV and cisplatin in triple-negative breast cancer. Breast Cancer Res. 2014;16:435.

13. Jasinski-Bergner S, Mandelboim O, Seliger B. The role of micrornas in the control of innate immune response in cancer. JNCI J Natl Cancer Inst. 2014;106:dju257-7.

14. Shah NR, Chen H. MicroRNAs in pathogenesis of breast cancer: implications in diagnosis and treatment. World J Clin Oncol. 2014;5:48-60.

15. Pogribny IP, Filkowski JN, Tryndyak VP, Golubov A, Shpyleva SI, Kovalchuk O. Alterations of microRNAs and their targets are associated with acquired resistance of MCF-7 breast cancer cells to cisplatin. Int J Cancer. 2010:127:1785-94.

16. Cascione L, Gasparini P, Lovat F, Carasi S, Pulvirenti A, Ferro A, et al. Integrated microRNA and mRNA signatures associated with survival in triple negative breast cancer. PLoS One. 2013;8:e55910.

17. Buffa FM, Camps C, Winchester L, Snell CE, Gee HE, Sheldon H, et al. microRNA-associated progression pathways and potential therapeutic targets identified by integrated mRNA and microRNA expression profiling in breast cancer. Cancer Res. 2011;71:5635-45.

18. Volinia S, Croce CM. Prognostic microRNA/mRNA signature from the integrated analysis of patients with invasive breast cancer. Proc Natl Acad Sci U S A. 2013;110:7413-7.

19. Garcia Al, Buisson M, Bertrand P, Rimokh R, Rouleau E, Lopez BS, et al. Down-regulation of BRCA1 expression by miR-146a and miR-146b-5p in triple negative sporadic breast cancers. EMBO Mol Med. 2011;3:279-90.

20. Kumaraswamy E, Wendt KL, Augustine L a, Stecklein SR, Sibala EC, Li D, et al. BRCA1 regulation of epidermal growth factor receptor (EGFR) expression in human breast cancer cells involves microRNA-146a and is critical for its tumor suppressor function. Oncogene. 2015; 34:4333-4346.

21. Kawai S, Amano A. BRCA1 regulates microRNA biogenesis via the DROSHA microprocessor complex. J Cell Biol. 2012;197:201-8.

22. McShane LM, Altman DG, Sauerbrei W, Taube SE, Gion M, Clark GM REporting recommendations for tumor MARKer prognostic studies (REMARK). Breast Cancer Res Treat. 2006;100:229-35.

23. van Beers EH, Joosse SA, Ligtenberg MJ, Fles R, Hogervorst FBL, Verhoef S, et al. A multiplex PCR predictor for aCGH success of FFPE samples. $\mathrm{Br}$ J Cancer. 2006;94:333-7.

24. Esteller M, Silva JM, Dominguez G, Bonilla F, Matias-Guiu X, Lerma E, et al. Promoter hypermethylation and BRCA1 inactivation in sporadic breast and ovarian tumors. J Natl Cancer Inst. 2000;92:564-9.

25. Boukerroucha $M$, Josse $C$, Segers $K$, El-Guendi S, Frères $P$, Jerusalem $G$, et al BRCA1 germline mutation and glioblastoma development: report of cases. BMC Cancer. 2015;15:181 
26. Marée R, Stévens B, Rollus L, Rocks N, Lopez X, Salmon I, et al. A rich internet application for remote visualization and collaborative annotation of digital slides in histology and cytology. Diagn Pathol. 2013;8 Suppl 1:S26

27. Dvinge H, Git A, Gräf S, Salmon-Divon M, Curtis C, Sottoriva A, et al. The shaping and functional consequences of the microRNA landscape in breast cancer. Nature. 2013;497:378-82.

28. Al-Mulla F, Abdulrahman M, Varadharaj G, Akhter N, Anim JT. BRCA1 gene expression in breast cancer: a correlative study between real-time RT-PCR and immunohistochemistry. J Histochem Cytochem. 2005;53:621-9.

29. Pérez-Vallés A, Martorell-Cebollada M, Nogueira-Vázquez E, García-García JA, Fuster-Diana $\mathrm{E}$. The usefulness of antibodies to the BRCA1 protein in detecting the mutated BRCA1 gene. An immunohistochemical study. J Clin Pathol. 2001;54:476-80.

30. Lips EH, Laddach N, Savola SP, Vollebergh MA, Oonk AMM, Imholz ALT, et al. Quantitative copy number analysis by multiplex ligation-dependent probe amplification (MLPA) of brca1-associated breast cancer regions identifies brcaness. Breast Cancer Res. 2011:13:R107.

31. Erturk E, Cecener G, Tezcan G, Egeli U, Tunca B, Gokgoz S, et al. BRCA mutations cause reduction in miR-200c expression in triple negative breast cancer. Gene. 2015;556:163-9.

32. Liang T, Guo L, Liu C. Genome-wide analysis of mir-548 gene family reveals evolutionary and functional implications. J Biomed Biotechnol. 2012;2012:679563.

33. Piriyapongsa J, Jordan IK. A family of human microRNA genes from miniature inverted-repeat transposable elements. PLoS One. 2007;2:e203.

34. Li Y, Ma X, Zhao J, Zhang B, Jing Z, Liu L. microRNA-210 as a prognostic factor in patients with breast cancer: meta-analysis. Cancer Biomark. 2013:13:471-81.

35. Bovy N, Blomme B, Frères $\mathrm{P}$, Dederen $\mathrm{S}$, Nivelles $\mathrm{O}$, Lion $\mathrm{M}$, et al. Endothelial exosomes contribute to the antitumor response during breast cancer neoadjuvant chemotherapy via microRNA transfer. Oncotarget. 2015;6:10253-66.

36. Frères $P$, Josse $C$, Bovy $N$, Boukerroucha $M$, Struman I, Bours $V$, et al. Neoadjuvant chemotherapy in breast cancer patients induces mir-34a and mir-122 expression. J Cell Physiol. 2015;230:473-81.

37. Lips EH, Mulder L, Oonk A, van der Kolk LE, Hogervorst FBL, Imholz ALT, et al. Triple-negative breast cancer: BRCAness and concordance of clinical features with BRCA1-mutation carriers. Br J Cancer. 2013;108:2172-7.

38. Vlachos IS, Kostoulas N, Vergoulis T, Georgakilas G, Reczko M, Maragkakis M, et al. DIANA miRPath v.2.0: investigating the combinatorial effect of microRNAs in pathways. Nucleic Acids Res. 2012;40(Web Server issue):W498-504.

39. Li JH, Liu S, Zhou H, Qu LH, Yang JH. StarBase v2.0: decoding miRNA-ceRNA, miRNA-ncRNA and protein-RNA interaction networks from large-scale CLIP-Seq data. Nucleic Acids Res. 2014:42:92-7.

\section{Submit your next manuscript to BioMed Central and take full advantage of:}

- Convenient online submission

- Thorough peer review

- No space constraints or color figure charges

- Immediate publication on acceptance

- Inclusion in PubMed, CAS, Scopus and Google Scholar

- Research which is freely available for redistribution 